\title{
The Development of Digital Technology in Radio Industry
}

\author{
Jieru Zhang* \\ Communication University of China \\ Beijing, P.R.China 100024
}

\begin{abstract}
This paper has explored the rise of digital radio broadcasting services and has discussed how these new DAB services have been integrated into the domain of traditional broadcasting and the wider media industry. And this study delineated how the broadcaster and listener have changed their roles in the digitization process. And discuss the fact that Internet radio services have broadened the scope of radio as a medium and provided new ways for listeners to access their favourite content and explore new possibilities.
\end{abstract}

Keywords-DAB (Digital Audio Broadcasting); terrestrial broadcasting; internet radi; radio listeners

\section{INTRODUCTION}

As developments in digital technology permeate the lives of people, many types of media are adopting digital technology across a wide range of industries, particularly the radio industry. Radio is a popular medium that is underscored by its prevalent usage. At an estimated 967 hours per year per person, radio is second only to the 1580 hours per person spent watching television and is more than six times the hours spent reading newspapers (Compaineb \& Smith, 2001). Over the last few decades, Frequency Modulation (FM) broadcasts have become popular among listeners. FM broadcast has the best sound and the broadcast quality is appreciated by regular listeners. However, the main problem with this analogue narrowband transmission method is the lack of resistance to multipath propagation, in particular, reception in mobile phones since this uses frequency-selective and time-selective radio channels. Furthermore, the multipath propagation fading phenomenon can have an adverse effect on reception quality. Although the use of a number of new methods and technologies to improve broadcast quality have been somewhat successful, there are some limitations to their application as they do not completely change the inherent weaknesses of FM broadcasting. In addition, due to the rise in income levels and cultural living standards, service users now demand higher quality broadcast services. Therefore, digital technology has been introduced to the media industry. In light of this, the following paper will discuss the digital broadcasting industry in terms of terrestrial broadcasting and Internet communication. In addition, this study will delineate how the broadcaster and listener have changed their roles in the digitisation process.

\section{THE Rise OF Digital TECHNOLOGY}

There is no specific definition of digital radio as it is quite an obscure notion. In modern day society, the majority of households have access to digital TV channels via cable, satellite or terrestrial transmissions and typically have access to a selection of radio stations as part of these service packages. Thus, one form of digital radio service allows people to listen to the radio via their television set. The second form of service allows people to listen to radio streams online (Lax, et al., 2010).

For the purposes of this study, we define digital radio as the transmission path of broadcast radio that uses digital signals instead of analogue signals. This practice is the key difference between digital radio and analogue radio receivers and digital radio production systems as these do not require a new receiver in order to function. However, digital radio transmissions do require the service user to buy additional receiving equipment in order to decode what is being transmitted.

\section{A. DAB as Terrestrial Broadcasting}

Commonly known as DAB, Digital Audio Broadcasting refers to a new form of broadcasting technology that is transmitted from either a ground station or via satellite in a similar way to conventional AM FM broadcast systems O'Neill, 2009). DAB provides a high calibre service and has revolutionised the digital broadcasting industry by transmitting data through a data signal, a process referred to as digital multimedia broadcasting. This broadcasting method uses channel coding, source coding, modulation and synchronisation network technology, which enables DAB to prevent fading induced by multi-channel propagation. Thus, $\mathrm{DAB}$ is capable of providing fixed and mobile reception that is of a similar quality to $\mathrm{CD}$ audio. This system also allows for the more effective use of radio resources as DAB can transmit multiple sets and multimedia digital sound programme services at the same time. DAB has already been adopted in many different parts of the world; for instance, a trial or operational version of DAB has been implemented in Australia, Belgium, Canada, Finland, France, Germany, India, Italy, Norway, Sweden and Switzerland. In fact, many European countries have achieved exceptional coverage rates with the UK having achieved $90 \%$ penetration. In 2014, a report published by Ofcom entitled 'The Communications

*Ph.D. of Art and Science. Communication University of China 
Market: Digital Radio Report' revealed that $48.5 \%$ of people in the UK currently own a DAB digital radio set. In addition, this report discovered that $54.8 \%$ of newly manufactured cars contain DAB digital radio sets. Thus, according to Ofcom (2014), the majority of radio listeners use DAB sets to listen to the radio.

DAB was designed as an alternative to FM that is suitable for fixed radio, portable and mobile receptions. The strength and quality of this system enables it to effectively transmit audio and data transmissions. In order to convey data along with an audio programme, programme associated data (PAD) is employed and integrated into the digital audio bitstream at a capacity of $667 \mathrm{bits} / \mathrm{s}$ to $65 \mathrm{kbits} / \mathrm{s}$. For example, DLS (Dynamic Label Segment) is used to display song information and MOT (Multimedia Object Transfer) is used to create slideshows with visual information relating to each artist/song. Furthermore, as a distinct dedicated data service, NPAD (Non-programme Associated Data) can also be transmitted. For instance, common NPAD services include weather reports, traffic news, flight data, news images and video clips (Bagharib \& Tan, 2005).

Throughout many European countries, such as the UK, Canada, Singapore, Australia, Belgium, Canada, China, Finland, Denmark, France, Germany, India, Italy, Norway, Taiwan, Sweden and Switzerland, commercial and trial versions of DAB services have been implemented. In fact, it is estimated that at least 300 million people worldwide can now access almost 600 different digital radio services using Eureka-147 DAB. On the market, there are a wide variety of DAB receivers with the most cost-effective models starting at US\$95. To date, at least 1.5 million DAB-enabled receivers, including PCI cards, CD players, clock radios, boom boxes, midi-hifi systems, home tuners, kitchen radios and portable hand-held devices, have been purchased (Select Committee on Communications, 2010).

Nonetheless, there are still limitations to the DAB system, particularly in relation to the technology it employs. More specifically, DAB, HD radio and DRM have not advanced the functional capacity of radio broadcasting systems beyond that which is offered by traditional analogue systems. Thus, many broadcasters are reluctant to embrace new digital systems when they are unlikely to yield a positive return on their investment, particularly when the cost of new equipment is taken into account. As a result of these limitations, many service users are reluctant to upgrade to a DAB system. Interestingly, a higher level of reluctance has been experienced in regions that have achieved the most progress in digital broadcasting.

The scope of DAB is also limited by the fact that system designers formulated new technology that would suit the needs of radio broadcasters who tend to offer digital variations of conventional methods as opposed to endorsing the innovative development of digitalisation and convergence. In most cases, mangers of media channels have chosen to ignore the implications of this issue by instead allowing the most powerful broadcasting bodies, from both an economic and political point of view, to oversee the transition in a way that bests suits their own interests.
Nonetheless, the convergence phenomenon has led to the creation of many new digital radio forms, including online streaming services and satellite audio services. Furthermore, many of these new radio forms have challenged the notion of radio and how it can be defined from the perspective of listeners.

These limitations highlight the main issue preventing the full realisation of DAB potential. More specifically, if digital broadcast systems cannot offer a medium through which conventional analogue operations can be performed in the cultivation of a convergent media environment, how is the radio likely to develop in the future? Although many media regulators are keen to achieve full digitalisation of their services, broadcasters and service users are reluctant to abandon analogue processes. However, those who promulgate convergence as the inevitable end-state of media systems argue that the full transition from analogue to digital is simply unavoidable (Anderson, 2012).

\section{B. The Development of Internet Radio}

The development of digital broadcasting systems has had a significant effect on radio as a medium, particularly in terms of how radio is created, delivered and purchased by service users. In effect, radio has become far more complex in providing many additional non-audio based services, including the provision of metadata, synchronised slideshows and video clips. Thus, radio services are no longer linear transmissions from emission mast to end user as audio files can now be accessed immediately or stored on a local level to facilitate time-shifted playouts. Furthermore, radio services are now more accessible and convenient for the listener as they are no longer obliged to adhere to the schedule implemented by the broadcaster.

Internet radio (IR) has only been developed in the past number of decades but has risen to prominence in recent years as one of the most fundamental distribution channels of audio streams and files. According to Kozamernik and Mullane (2005), based on audience figures, IR is now frequently used in office environments and is the medium of choice for young radio listeners.

An audio service that is transmitted using online resources is known as Internet radio and radio services broadcast within this domain are generally known as webcasting as these services are not generally transmitted wirelessly. These services allow the user to access a constant audio stream that cannot typically be paused or replayed. In this sense, Internet radio resembles more conventional broadcast mediums. In addition, the inability to pause or replay content distinguishes Internet radio services from ondemand file services and podcasts, where content is usually downloaded as opposed to streamed. Radio services offered online cover a wide range of genres, including news, sports, discussion, and many different types of music. In effect, these services offer the same range of formats as traditional broadcast radio channels. While many Internet radio channels are not affiliated with a terrestrial radio station, many services are offered in conjunction with a traditional radio network. The increasing number of independent online 
stations is of course attributable to the minimal start-up and operational costs of running an Internet radio service (Evansfm, 2014).

Almost one billion people across the world have access to an internet connection with $70 \%$ of people in the US having internet connections at home and $30 \%$ having internet availability at their place of work. Similarly, approximately $60 \%$ of people in South Korea, Japan, Canada and Germany have internet connections. Thus, it is clear that the internet coverage rate is rising rapidly. In fact, it has been reported that a total of 31 new internet connections are made each month with each user spending an average of 26 hours browsing 66 different websites and 1,268 different webpages each month. Of those with internet access, $87 \%$ use the service to send emails, $55 \%$ use it to send files and $60 \%$ use it to send instant messages. According to reports, $22 \%$ of internet users have used online video services.

Arbitron/Edison, a media research company in the US, conducted a large-scale study on the use of Internet services and Multimedia in the US in 2005 (www.arbitron.com). The findings indicate that a total of 55 million people access Internet radio and video services every month. Based on the same study, there are several reasons why people choose to listen to Internet radio as opposed to traditional radio stations:

- To access audio that is unavailable elsewhere

- To choose what music is played

- To avoid commercial breaks

- To listen to a more diverse range of musical genres

- To obtain a better quality signal

- To avoid excessive chatter amongst presenters

- To embrace technological change

Of those that listen to Internet radio, the majority listen to several well-known brands, such as America Online's AOL Radio Network, Yahoo! Music, Microsoft's MSN Radio, WindowsMedia.com and Live365. Between 06:00 and 24:00, each of these stations has an average of 4.8 million listeners over the age of 12 years. According to Kozamernik and Mullane (2005), 25\% of Internet radio listeners listen to one of these five renowned Internet radio brands in the US (Kozamernik \& Mullane, 2005).

\section{1) Characteristics of Internet Radio}

There are three key differences between radio transmitted via the Internet and other forms of delivery media:

a) Internet radio is a comparatively recent phenomenon that offers listeners a new way of listening to radio services using a computer device. This requires the use of a different interface format, including a mouse, computer screen and keyboard, and enables the user to look through different radio content options based on location, style or genre. In addition, they can opt for the 'now playing' option. In this case, the frequency, either AM or $F M$, or multiplex, generally DAB, of the station's frequency is largely unimportant. The listeners can also create customised favourites lists and create a unique virtual station based on their individual preferences. An 'ondemand' service is generally provided by traditional broadcasters where users can access and play a range of archived content that has recently been broadcast on traditional channels.

b) The number of service providers is far more expansive on Internet radio as providers include traditional broadcasters, portals, online-based broadcasters and unaffiliated users.

c) The range of content on Internet radio is far more diverse as there are a huge number of stations that specialise in any number of formats and genres. Thus, listeners can select from the most popular stations or search for stations that are playing specific songs that are currently in the charts. In addition, users can time-shift the play-out as computers employ hard disc memory (Evenis, 2006).

2) The Relationship between Internet Radio and Traditional Radio

The ease with which service providers can enter the Internet radio market has led to a substantial increase in the number of Internet radio stations available. Thus, it is now more important than ever for each site to market their services so that they achieve a stronger competitive advantage. Generally speaking, most public service broadcasters are the most popular online radio sites as they have already established a positive brand image and can access the listener across a diverse range of mediums, including radio, television and the Internet.

Broadcasters must market their online services effectively in order to secure a loyal online listener base. Although the marketing techniques of online broadcasters are beyond the scope of this paper, it is important to highlight that effective marketing can be achieved using a range of different strategies. For instance, an Internet radio station can promote their services using online advertising campaigns, press releases, on-air giveaways, print advertisements or email marketing strategies. However, the concept of Internet radio is a marketing mechanism in itself as users must avail of the service before deciding whether or not to embrace the concept and become avid listeners of online broadcasting content. Many traditional broadcasters, such as the BBC, have discovered that the provision of Internet radio services can have a positive effect on listenership figures for traditional radio broadcasts. In fact, Internet radio services can encourage listeners to explore a range of different programmes. Furthermore, many shows have an equal number of 'catch-up' listeners that listen to radio programmes online after they have been broadcast live (Kozamernik \& Mullane, 2005).

The BBC promotes its Internet radio player by offering links to the most popular shows and providing lists of the available content based on topic or genre. In addition, future versions of this online platform will offer suggestions to the user based on what they have already listened to. In effect, the radio station will use the 'if you liked that, you may also 
enjoy this' feature that is common to websites such as Amazon and Q-Magazine. Nonetheless, in its current format, the BBC's online player is claimed to have increased listenership figures by millions.

Internet radio sites can also be used to forge online communities of dedicated listeners and to perform quantitative research into the habits of listeners. This can be achieved by offering a forum on the radio website in which users can interact with one another and discuss radio content. This strategy would also enable the radio station to collect demographical data on each user as they would be required to register their details in order to participate in the online community. This information can then be used when planning marketing campaigns.

\section{2.3 The Evolution of the Radio Broadcaster}

The radio industry is now focused on becoming a more multimedia web-based business by enhancing coverage, branding and radio content. These measures also relate directly to the creation of a business structure that complements the framework of the radio industry and satisfies the needs of broadcasters and their complex production process (Hesmondhalgh, 2002). In addition, as radio content is largely intangible, it falls within the realm of cultural industries and is thus reliant upon the distinction of different user demographics. To achieve this, many radio stations market themselves to specific user groups (Nyre, 20008). The level of coverage provided also has a strong impact on branding and content as branding is reliant on content and content is reliant on coverage. In effect, in terms of spectrum distribution, a broadcaster must adhere to the broadcasting regulations in different countries and will often determine how much coverage to offer based on the listenership figures within different countries/regions. By offering widespread coverage, the broadcaster ensures that all content is accessible to those with an internet connection and this can help cultivate and maintain a strong brand image. In other words, the ease with which users can access content will often determine the long-term success of a radio station. Recent figures also suggest that online radio is becoming more popular than FM radio, particularly with young people and immigrants; nonetheless, terrestrial broadcasters still acquire the highest number of listeners (Cordeiro, 2012).

Online radio branding typically involves the use of branding strategies that are commonly employed within all realms of the media industry. In the past, marketing strategies focused on promoting programme content in response to the increasing popularity of television during the 1950s. The rise of television viewership figures had a serious effect on radio development as marketers were forced to segment the market in order to tailor and market services toward specific user demographics. For instance, during the 1960 s, the car radio and the transistor radio increased the popularity of radio content amongst drivers and the youth demographic. Over time, the marketing campaigns for radio stations became more professional as SWOT analysis was performed to determine the role played by radio in the media industry. The most popular broadcasters in Portugal have a robust management structure with different departments taking charge of different aspects of the station, such as programming, music, marketing, multimedia, sales and broadcasting personalities. Thus, the role of management is to create a strong station identity that facilitates dialogue and user interaction and becomes synonymous with different lifestyles or social groups. In the present day, radio stations have cultivated brand communities as the emotional or intangible meanings associated with radio brands are more valuable than its basic functional qualities (Cordeiro, 2012). In other words, niche stations have begun to acquire cult status (Ragas and Bueno, 2001) as listeners identify with radio brands that complement their interests and feel as if they belong to a wider community of radio listeners that share their perspectives on life, music, society etc.

Thus, radio content is directly influenced by this marketing process and a brand is considered dependable when they succeed in satisfying the needs of the consumer. In light of this, radio management must offer high quality content and renowned broadcasting personalities that appeal to the target market and must create a sense of belonging among users so that they feel as if they form part of wider radio-based community.

\section{New Digital Radio Listeners}

The main objective of the media is to initiate a communication process by offering new perspectives or outlooks on specific issues in order to stimulate two-way discourse. However, the media also aims to make full use of the network and the valuable resources it provides. From another perspective, a high degree of interactivity can stimulate group dialogue and create new virtual communities in a similar way to those created on Facebook (Cordeiro, 2011). While the number of 'likes' does not indicate the extent of user interaction with radio content, the "page insights' feature can be useful in determining the effectiveness of a page. Using this strategy, it is clear that Rádio Comercial currently accesses at least 58,000 people while 80,000 people discuss posts made by RFM. In other words, these pages generated a high level of community interaction via comments or sharing.

The extent to which online listeners interact can be determined based on social network comments, emails, blog posts, images, audio or video. Radio stations generate multimedia content and generate different narratives across a variety of platforms in order to encourage a higher level of listener participation. For instance, online radio stations often provide live streaming services, audio podcasts, on-demand video/audio and social media content. In the past, interactivity was directly linked to the various forms of listener participation, such as letters, competitions or telephone calls. On a digital level, the forms of participation have grown increasingly diverse as users can interact without necessarily having to communicate directly with the broadcaster. Nonetheless, the provision of more customisable features increase user control and participation and increase the level of listener interactivity accordingly (Cordeiro, 2012). 
The average FM and online radio listener is an avid web user with an interest in technology that listens to online radio for convenience when they are unable to listen to FM radio. This individual also participates in social media but updates their profile infrequently. According to Cordeiro (2011a), this individual is also quite lazy in terms of online searches as they claim to not have time to search for content more effectively.

These average user descriptions facilitate the analysis of user consumption habits in terms of online radio and traditional broadcasting services. These descriptions also highlight the different habits of different demographics and the varying preferences of different target audiences (Cordeiro, 2012)The most significant aspect of the e-listener is that they listen to the radio in conjunction with other types of audio media. In other words, while listeners often choose radio stations based on what is available on different platforms, they are generally more swayed by content as opposed to media channels. Thus, if a listener is an avid follower of a particular brand, they are likely to listen to this brand using the audio platform that is most convenient.

Thus, the e-listener is essentially a multimedia crossplatform user that demonstrates interactive tendencies when using all media formats and platforms and accessing all types of media content.

Research also shows that radio listeners now visit multimedia radio station websites as well as listen to FM broadcasts. Thus, it is not so much the radio content that has changed but the ways in which it can be accessed. However, while there are several media platforms available, the user's final choice of medium will depend on their own needs and preferences. Nonetheless, the majority of listeners now use a wide variety of platforms interchangeably based on their location and available resources.

Studies have shown that more and more listeners take a multimedia approach to radio consumption as they access web-based audio content and interact with other users via social media platforms to discuss content and share recommendations. In addition to radio content, these users often access podcasts created by radio stations and users, online music playlists, web-radios, music communities and music portals (Cordeiro, 2011b).

Users can also communicate with the station using a diverse range of methods and are no longer forced to rely on conventional means. This cultivates the illusion of a radio community in which two-way dialogue is achieved (Criswell, 1994). During the 1970s, McLuhan and Nevitt claimed that technological advances would transform the user into a producer and were correct in making this prediction as many users now play an active role in the consumer culture in creating and sharing experiences, exchanging knowledge and establishing vast online networks amongst imagined communities. Nonetheless, there are still a large number of passive consumers who have little interest in finding and modifying media sources to suit their requirements. In summary, a high level of integration is achieved by creating virtual communities based on the mechanisms that enable listeners to engage with and participate in online radio.

\section{CONCLUSION}

In conclusion, this paper has explored the rise of digital radio broadcasting services and has discussed how these new $\mathrm{DAB}$ services have been integrated into the domain of traditional broadcasting and the wider media industry. More specifically, this essay has demonstrated how digital broadcasting methods are being employed by existing broadcasters as well as independent users to diversify the scope of current radio services and increase listenership figures by providing a broader range of multimedia services across a broad range of audio platforms. However, while digital radio services have grown increasingly popular on account of their convenience, they operate alongside traditional analogue services and are not designed as a replacement. In other words, Internet radio services have broadened the scope of radio as a medium and provided new ways for listeners to access their favourite content and explore new possibilities. Thus, the rise of digital radio does not imply that analogue methods should be abandoned, it simply implies that radio as a medium must continue to evolve in line with the needs and demands of the consumer. In effect, rising income levels and standards of living have led consumers to demand radio content that is both accessible and of the highest quality. The development of Internet radio has had a positive effect on radio listenership figures, particularly for traditional broadcasters that now offer online streaming facilities. Thus, broadly speaking, broadcasters and listeners have played an active role in the development of digital radio as stations cultivate a community of service users who establish a sense of rapport with other listeners. This transition mirrors the development of virtual communities on social media platforms and indicates how radio broadcasters can maintain their listenership base by engaging with the listener in an online environment and providing additional non-audio services to acquire a competitive advantage and disseminate a positive brand image for the station.

\section{REFERENCES}

[1] Anderson, J. N., 2012. Radio broadcasting's digital dilemma Convergence: The International Journal of Research into New Media Technologies, Issue 1354856512451015.

[2] Bagharib, A. \& Tan, R., 2005. Digital Radio Revolution - The Future

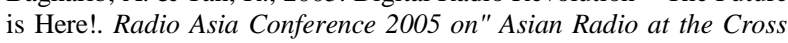
Roads: A New Paradigm in the Digital Age, Volume 15.

[3] Castells, M., 2011. The Rise Of The Network Society, The Information Age: Economy, Society And Culture. s.1.:John Wiley \& Sons.

[4] Cordeiro, P., 2011b. Generations and Online Media: The Study of Baby Boomers, Generation X and Generation Y Relation With Radio. Media+Generations Conference Proceedings Book, Milan: Peter Lang, pp. 123-143.

[5] Cordeiro, P., 2011. Hello Facebookers! Radio in social networks: how do radio stations, radios hosts and listeners engage through Facebook?. Available at: http://netfm.wordpress.com/2011/04/09/hellofacebookers [Accessed 0905 2015].

[6] Cordeiro, P., 2011a. Reconceptualizing Audience Research and Survey Strategies for Radio: Portuguese Case. Iletisme: arastirmalari, 5(1), pp. 75-101. 
[7] Cordeiro, P., 2012. Radio becoming r@dio: Convergence, interactivity and broadcasting trends in perspective. Participations, 9(2), pp. 492-510

[8] Crisell, A., 1994. Understanding Radio. second editor ed. London: Routledge.

[9] Compaine, B., \& Smith, E., 2001. Internet radio: A new engine for content diversity?. arXiv preprint cs/0109046.

[10] Evansfm, 2014. What is radio?. [Online] Available at: http://www.evansfm.co.uk/what-is-radio. [Accessed 0705 2015].

[11] Evenis, I. G. G. A., 2006. Audio on demand: Radio's future format and its impact on the communication procedure. [Online] Available

at: https://www.academia.edu/306235/Audio_on_demand Radio_s futur e format and its impact on the communication procedure [Accessed 0805 2015]

[12] Hesmondhalgh, D., 2002. The Cultural Industries. LONDON: Sage.

[13] Kozamernik, F. \& Mullane, M., 2005. An introduction to Internet radio. EBU Technical Review, Geneva.

[14] Lax, S., Ala-Fossi, M. \& Per Jauert, H. S., 2010. DAB: the future of radio? The development of digital. Digital Radio in Europe: Technologies, Industries and Cultures, Bristol: Intellect, pp. 99-119.

[15] Nyre, L., 2008. Sound Media, From Live Journalism To Musical Recording. London: Routledge.

[16] O'Neill, B. (2009). DAB Eureka-147: a European vision for digital radio. New Media \& Society, 11(1-2), pp. 261-278.

[17] Ragas, M. W. B. B. J., 2002. The Power Of Cult Branding. New York: Crown Business.

[18] Select Committee on Communications, 2010. Digital switchover of television and radio in the United Kingdom, London: Authority of House of Lord.

[19] The Office of Communication, 2014. The Communications Market: Digital Radio Report, Ofcom's fifth annual digital progress report, s.l.: s.n.

[20] WorldDAB Forum website, 2014. WorldDAB Forum website. [Online] Available at: http://www.worlddab.org/country-information [Accessed 0505 2015]. 